\title{
Flap endonuclease 1 polymorphisms (rs174538 and rs4246215) contribute to an increased cancer risk: Evidence from a meta-analysis
}

\author{
HONGTAO REN ${ }^{1 *}$, HONGBING MA ${ }^{1 *}$, YUE KE $^{1}$, XIAOBIN MA $^{1}$, DAN XU $^{2}$, \\ SHUAI LIN ${ }^{1}$, XIJING WANG ${ }^{1}$ and ZHI-JUN DAI ${ }^{1,2}$ \\ ${ }^{1}$ Department of Oncology, The Second Affiliated Hospital of Xi'an Jiaotong University, Xi'an, Shaanxi 710004; \\ ${ }^{2}$ Center for Translational Medicine, Frontier Institute of Science and Technology, \\ Xi'an Jiaotong University, Xi'an, Shaanxi 710028, P.R. China
}

Received February 3, 2015; Accepted February 26, 2015

DOI: $10.3892 / \mathrm{mco} .2015 .617$

\begin{abstract}
Flap endonuclease-1 (FEN1) is a key factor during the maintenance of genomic stability and protection against tumorigenesis. Since the identification of functional polymorphisms of FEN1 (rs174538 and rs4246215), numerous studies have evaluated the association between the two single-nucleotide polymorphisms and cancer risk. To derive a more precise estimation, a meta-analysis was performed on the association between the FEN1 polymorphisms (rs174538 and rs4246215) and cancer risk. Odds ratios (ORs) and 95\% confidence intervals (CIs) were used to estimate the strength of the associations. Thirteen case-control studies, including 5,108 cases and 6,382 case-free controls, were identified. For rs174538, individuals with the GG or GA genotype had an increased risk of cancer when compared to the -69AA genotype (AA vs. GG: OR, 1.85; 95\% CI, 1.65-2.08; P<0.00001; AA vs. GA: OR, 1.43; 95\% CI, 1.27-1.60; P<0.00001; AA vs. GG+GA: OR, 1.28; 95\% CI, 1.16-1.42; $\mathrm{P}<0.00001)$. For rs4246215, similar results were identified, as the GG or GT genotype was significantly associated with the increased cancer risk when compared to TT (TT vs. GG: OR, 1.71; 95\% CI, 1.52-1.92; P<0.00001; TT vs. GT: OR, 1.34; 95\% CI, 1.20-1.50; P<0.00001; TT vs. GG+GT: OR, 1.50; 95\% CI, 1.35-1.67; P<0.00001). The present meta-analysis indicated that FEN1 rs174538 and rs4246215 polymorphisms may contribute to an increased risk of cancer.
\end{abstract}

Correspondence to: Dr Zhi-Jun Dai, Department of Oncology, The Second Affiliated Hospital of Xi'an Jiaotong University, 157 Xiwu Road, Xi'an, Shaanxi 710004, P.R. China

E-mail: dzj0911@126.com

*Contributed equally

Key words: FEN1, single-nucleotide polymorphism, cancer, meta-analysis

\section{Introduction}

Flap endonuclease 1 (FEN1) is a versatile, structure specific and multifunctional nuclease involved in DNA replication and repair $(1,2)$. Human FEN1, which is the archetypal member of the $\operatorname{Rad} 2$ nuclease family $(3,4)$, is located on chromosome 11q12 and consists of two exons and one intron. FEN1

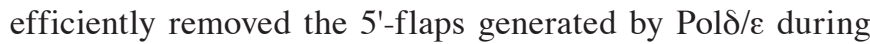
repair synthesis of long-patch base-excision repair (LP-BER) and removed primers during lagging-strand DNA synthesis and Okazaki fragment processing $(3,5,6)$. Furthermore, FEN1 can be stimulated to promote apoptotic DNA fragmentation following apoptotic stimuli, acting as a 5' exonuclease (1) and a gap-dependent endonuclease $(7,8)$, as reported via its ability to participate in multiple protein-protein interactions. Thus far, $>30$ FEN1-interacting proteins have been identified (2). Of these FEN1 interaction partners, proliferating cell nuclear antigen (PCNA), which was initially identified as a replication accessory protein, accompanies FEN1 in all FEN1-involved DNA metabolic pathways except for the apoptotic DNA fragmentation pathway, suggesting a critical role of the FEN1/PCNA interaction in regulating LP-BER (9). A tumor suppressor function for FEN1 has been shown in preclinical models (10-14). Therefore, FEN1 has been considered as a key factor during maintenance of genomic stability and protecting against carcinogenesis.

However, being a multifunctional factor, mutation of FEN1 has been suggested to cause genomic instability and predisposition to cancer. The functional impairment of yeast RAD27 (the homolog of mammalian FEN1) leads to a marked increase in the rate of spontaneous mutations $(8,15,16)$. A recent study showed that groups of FEN1 mutations in cancer specimens that abrogated two of the three nuclease activities lead to cancer initiation and progression (17). Yang et al (18) identified two single-nucleotide polymorphisms (SNP), -69G>A (rs174538, in the gene promoter region) and 4150G $>\mathrm{T}$ (rs4246215, in gene 3'-untranslated region), following thorough re-sequencing of the FEN1 locus in 30 Chinese Han healthy volunteers. The study identified that the $-69 \mathrm{G}>\mathrm{A}$ change leads to elevated promoter activity, which is most likely due to a higher binding affinity of the $\mathrm{G}$ allele with certain unknown transcriptional inhibitors. 
The $-69 \mathrm{G}>\mathrm{A}$ and $4150 \mathrm{G}>\mathrm{T}$ SNPs influenced gene expression in vivo subsequent to examining FENI mRNA in 38 lung normal tissues, 15 esophagus normal tissues, 12 stomach normal tissues and 13 normal tissues through quantitative analyses (18). Abnormal expression and/or function of FEN1 resulting from SNPs may possibly contribute to different cancer susceptibility. On the basis of the previous findings mentioned, we hypothesized that the functional genetic variants in the FENI gene may affect cancer risk. Meta-analysis is a statistical technique for combining results from different studies to produce a single estimate of the major effect with enhanced precision (19). Therefore, a meta-analysis of the published studies was conducted to derive a more precise estimation of the association between FEN1 polymorphisms and cancer risk.

\section{Materials and methods}

Identification and eligibility of relevant studies. Computer searches were carried out by two investigators independently in Embase, Pubmed, ISI Web of Knowledge and Chinese National Knowledge Infrastructure databases (until March 31, 2014) to collect case-control studies of the FENI SNPs (rs174538 and rs4246215) association with cancer risk. The keywords were as follows: Cancer/carcinoma, Flap endonuclease-1/FEN1, $-69 \mathrm{G}>\mathrm{A} / \mathrm{rs} 174538$ and $4150 \mathrm{G}>\mathrm{T} / \mathrm{rs} 4246215$ and polymorphism/genotype/SNP. In addition, reference lists of the main studies and reviews were also assessed by a manual search to identify additional relevant publications. The following criteria were used to select studies for further meta-analysis: i) Case-control studies; ii) studies that evaluated the association of FEN1 SNPs (rs174538 and rs4246215) on cancer risk; iii) studies that contained at least two comparison groups (cancer vs. control group); and iv) studies that included detailed genotyping data.

The following exclusion criteria were used accordingly: i) The design of the experiments were not case-control studies; ii) the source of cases and controls, and other essential information were not provided; iii) the genotype distribution of the control population was departure from Hardy-Weinberg Equilibrium; and iv) reviews and duplicated publications.

Data extraction. Evaluations of studies were performed independently by two investigators and data with discrepancies in identification were discussed by all investigators. For each included study, the following information was collected: First author, year of publication, country of origin, ethnicity, source of control, number of cases and controls, genotyping methods for rs174538 and rs4246215, total number of cases and controls, as well as number of cases and controls with $A / A, A / G, G / G$ and $\mathrm{T} / \mathrm{T}, \mathrm{T} / \mathrm{G}, \mathrm{G} / \mathrm{G}$ genotypes. All the case and control groups were well-controlled.

Statistical analysis. For the control group of each study, the allelic frequency was calculated. The strength of associations between FEN1 SNPs (rs174538 and rs4246215) and cancer risk were measured by odds ratio (OR) with $95 \%$ confidence interval (CI). For rs174538, the AA genotype was used as the reference genotype in all analyses. The risks of the GG and GA genotypes for cancers were estimated, compared to the AA homozygote, and subsequently the risks of GA+GG for cancer were evaluated, respectively. Accordingly, for rs 4246215 , the TT genotype

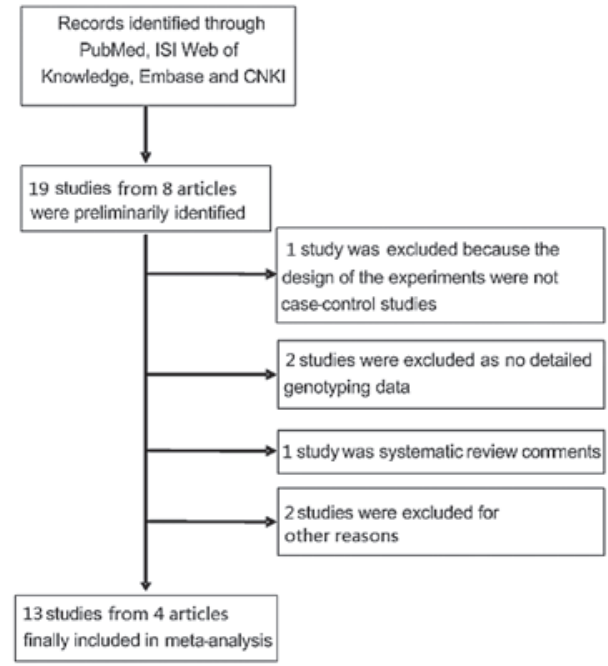

Figure 1. Flow chart of study selection.

was used as the reference genotype in all the analyses. The risks of the GT and GG genotypes for cancer were estimated, compared to the TT homozygote, and subsequently the risks of GT+GG for cancers were evaluated. The significance of the pooled OR was determined by the $\mathrm{Z}$ test. Statistical heterogeneity among studies was assessed with the $\mathrm{Q}$ and $\mathrm{I}^{2}$ statistics. The $\mathrm{Q}$ test and $\mathrm{I}^{2}$ were claimed to test the variation, which was due to heterogeneity or by random error. When the P-value of heterogeneity tests was $\mathrm{P} \leq 0.1$, the random effects model was used. When the P-value of heterogeneity test was $\mathrm{P} \geq 0.1$, the fixed effects model was used. Sensitivity analysis was also tested by removing one study at a time to calculate the overall homogeneity and effect size. Publication bias was evaluated by the funnel plot and further assessed by the method of Egger's linear regression test. All the statistical analyses were carried out with the Review Manager version 5.1 software (Revman; The Cochrane Collaboration, Oxford, United Kingdom). All $\mathrm{P}$-values in the meta-analysis were two-sided, and $\mathrm{P}<0.05$ were considered to indicate a statistically significant difference.

\section{Results}

Characteristics of studies. A total of 19 sub-studies from 8 studies that fulfilled our search criteria were preliminarily identified for further detailed evaluation (Fig. 1). One study was excluded as the designs of the experiments were not case-control studies. Two studies did not focused on FEN1 SNPs (rs174538 and rs4246215) and cancer risk. Two studies were excluded as there was no detailed genotyping data. Four studies were review comments. Finally, 13 sub-studies from 4 studies on rs174538 and rs4246215 genotypes and cancer risk were identified $(18,20-22)$, including a total of 5,108 cancer cases and 6,382 case-free controls. The characteristics of the included studies are listed in Table I. The included studies were all based on Chinese populations. All were case-control studies, including lung cancer, breast cancer, glioma, hepatocellular carcinoma, esophageal cancer, gastric cancer and colorectal cancer. All the types of cancer were confirmed by histology or pathology. Additionally, the controls were mainly matched on age, of which all the studies were hospital-based. 
Table I. Characteristics of the studies included in the meta-analysis.

\begin{tabular}{|c|c|c|c|c|c|c|}
\hline $\begin{array}{l}\text { First author, } \\
\text { year (Ref.) }\end{array}$ & Country & Ethnicity & $\begin{array}{l}\text { Cancer } \\
\text { type }\end{array}$ & $\begin{array}{l}\text { Genotyping } \\
\text { method }\end{array}$ & $\begin{array}{l}\text { Source of } \\
\text { control }\end{array}$ & $\begin{array}{c}\text { Total sample } \\
\text { size (case/control) }\end{array}$ \\
\hline \multicolumn{7}{|l|}{ Lv, 2014 (20) } \\
\hline 1 & China & Asian & Breast cancer & PCR-RFLP & Hospital & $800 / 800$ \\
\hline 2 & China & Asian & Breast cancer & PCR-RFLP & Hospital & $300 / 600$ \\
\hline Chen, 2013 (21) & China & Asian & Glioma & PCR-RFLP & Hospital & $317 / 802$ \\
\hline \multicolumn{7}{|l|}{ Liu, 2012 (22) } \\
\hline 1 & China & Asian & Hepatocellular carcinoma & PCR-RFLP & Hospital & $411 / 423$ \\
\hline 2 & China & Asian & Esophageal cancer & PCR-RFLP & Hospital & $266 / 386$ \\
\hline 3 & China & Asian & Gastric cancer & PCR-RFLP & Hospital & $220 / 250$ \\
\hline 4 & China & Asian & Colorectal cancer & PCR-RFLP & Hospital & $126 / 162$ \\
\hline 5 & China & Asian & Hepatocellular carcinoma & PCR-RFLP & Hospital & $237 / 315$ \\
\hline 6 & China & Asian & Esophageal cancer & PCR-RFLP & Hospital & $289 / 337$ \\
\hline 7 & China & Asian & Gastric cancer & PCR-RFLP & Hospital & $192 / 204$ \\
\hline 8 & China & Asian & Colorectal cancer & PCR-RFLP & Hospital & $110 / 145$ \\
\hline \multicolumn{7}{|l|}{ Yang, 2009 (18) } \\
\hline 1 & China & Asian & Lung cancer & PCR-RFLP & Hospital & $1,013 / 1,131$ \\
\hline 2 & China & Asian & Lung cancer & PCR-RFLP & Hospital & $827 / 827$ \\
\hline
\end{tabular}

PCR-RFLP, polymerase chain reaction-restriction fragment length polymorphism.

Table II. rs 174538 polymorphism genotype distribution and allele frequency in cases and controls.

\begin{tabular}{|c|c|c|c|c|c|c|c|c|c|c|c|c|}
\hline \multirow{3}{*}{$\begin{array}{l}\text { First author, } \\
\text { year (Ref.) }\end{array}$} & \multicolumn{8}{|c|}{ Genotype, n } & \multicolumn{4}{|c|}{ Allele frequency, n (\%) } \\
\hline & \multicolumn{4}{|c|}{ Case } & \multicolumn{4}{|c|}{ Control } & \multicolumn{2}{|c|}{ Case } & \multicolumn{2}{|c|}{ Control } \\
\hline & Total & GG & GA & AA & Total & GG & GA & AA & G & A & G & A \\
\hline Chen, 2013 (21) & 317 & 160 & 122 & 35 & 802 & 316 & 356 & 130 & 437 (69) & $197(31)$ & $988(62)$ & $616(38)$ \\
\hline \multicolumn{13}{|l|}{ Liu, 2012 (22) } \\
\hline 1 & 410 & 203 & 173 & 34 & 423 & 174 & 185 & 64 & $579(71)$ & $241(29)$ & $533(63)$ & $313(37)$ \\
\hline 2 & 266 & 137 & 105 & 24 & 386 & 163 & 168 & 55 & $379(71)$ & $153(29)$ & $494(64)$ & $278(36)$ \\
\hline 3 & 220 & 118 & 86 & 16 & 250 & 108 & 108 & 34 & $322(73)$ & $118(27)$ & $324(65)$ & $176(35)$ \\
\hline 4 & 126 & 64 & 51 & 11 & 162 & 65 & 73 & 24 & $178(71)$ & $73(29)$ & $203(63)$ & $121(37)$ \\
\hline 5 & 238 & 87 & 117 & 34 & 315 & 96 & 149 & 70 & $281(59)$ & $195(41)$ & $341(54)$ & $289(46)$ \\
\hline 6 & 289 & 107 & 144 & 38 & 336 & 100 & 163 & 73 & $358(62)$ & $220(38)$ & $393(54)$ & $309(46)$ \\
\hline 7 & 192 & 71 & 96 & 25 & 204 & 61 & 101 & 42 & $138(49)$ & $146(51)$ & $223(55)$ & $185(45)$ \\
\hline 8 & 110 & 40 & 53 & 17 & 145 & 44 & 71 & 30 & $133(60)$ & $87(40)$ & $159(55)$ & $131(45)$ \\
\hline \multicolumn{13}{|l|}{ Lv, 2014 (20) } \\
\hline 1 & 800 & 401 & 317 & 82 & 800 & 315 & 355 & 130 & $1,119(70)$ & $481(30)$ & $985(62)$ & $615(38)$ \\
\hline 2 & 300 & 146 & 120 & 34 & 600 & 200 & 284 & 116 & 412 (69) & $188(31)$ & $784(65)$ & $416(35)$ \\
\hline \multicolumn{13}{|l|}{ Yang, 2009 (18) } \\
\hline 1 & 1,013 & 505 & 402 & 106 & 1,131 & 467 & 496 & 168 & $1,402(70)$ & $614(30)$ & $1,430(63)$ & $832(37)$ \\
\hline 2 & 827 & 286 & 394 & 147 & 827 & 257 & 384 & 186 & $966(58)$ & $688(42)$ & $898(54)$ & $756(46)$ \\
\hline
\end{tabular}

Quantitative synthesis. The frequency of the A allele varied widely across the 13 studies, ranging from 23 to $46 \%$ in rs174538 among 6,381 healthy controls (Table II). The frequency of the $\mathrm{T}$ allele ranged from 35 to $46 \%$ in rs4246215 among 6,381 healthy controls (Table III). The average frequencies of the $\mathrm{A}$ and $\mathrm{T}$ allele in the two polymorphisms (rs174538 and rs4246215) were 39 and 41\%, respectively.

The main results of the meta-analysis are listed in Tables IV and V. Overall, there was evidence of an association between the variant genotypes and cancer risk in different genetic 
Table III. rs4246215 polymorphism genotype distribution and allele frequency in cases and controls.

\begin{tabular}{|c|c|c|c|c|c|c|c|c|c|c|c|c|}
\hline \multirow{3}{*}{$\begin{array}{l}\text { First author, } \\
\text { year (Ref.) }\end{array}$} & \multicolumn{8}{|c|}{ Genotype, n } & \multicolumn{4}{|c|}{ Allele frequency, n (\%) } \\
\hline & \multicolumn{4}{|c|}{ Case } & \multicolumn{4}{|c|}{ Control } & \multicolumn{2}{|c|}{ Case } & \multicolumn{2}{|c|}{ Control } \\
\hline & Total & GG & GT & $\mathrm{TT}$ & Total & GG & GT & TT & G & $\mathrm{T}$ & G & $\mathrm{T}$ \\
\hline Chen 2013 (21) & 314 & 160 & 120 & 34 & 802 & 309 & 363 & 130 & $440(70)$ & $188(30)$ & $981(61)$ & $623(39)$ \\
\hline \multicolumn{13}{|l|}{ Liu, 2012 (22) } \\
\hline 1 & 411 & 195 & 177 & 39 & 423 & 176 & 187 & 60 & $567(69)$ & $255(31)$ & $539(64)$ & $307(36)$ \\
\hline 2 & 249 & 115 & 114 & 20 & 386 & 161 & 172 & 53 & $344(69)$ & $154(31)$ & $494(64)$ & $278(36)$ \\
\hline 3 & 210 & 111 & 82 & 17 & 250 & 107 & 110 & 33 & $304(72)$ & $116(28)$ & $324(65)$ & $176(35)$ \\
\hline 4 & 119 & 61 & 47 & 11 & 161 & 65 & 74 & 22 & $169(71)$ & $69(29)$ & 204 (63) & $118(37)$ \\
\hline 5 & 237 & 85 & 118 & 34 & 315 & 98 & 148 & 69 & $288(61)$ & $186(39)$ & 344 (55) & $286(45)$ \\
\hline 6 & 289 & 110 & 141 & 38 & 337 & 101 & 164 & 72 & $361(62)$ & $217(38)$ & $366(54)$ & $308(46)$ \\
\hline 7 & 192 & 72 & 95 & 25 & 204 & 59 & 102 & 43 & $239(62)$ & $145(38)$ & $220(54)$ & $188(46)$ \\
\hline 8 & 110 & 39 & 55 & 16 & 145 & 44 & 71 & 30 & $133(60)$ & $87(40)$ & $159(55)$ & $131(45)$ \\
\hline \multicolumn{13}{|l|}{ Lv, 2014 (20) } \\
\hline 1 & 800 & 365 & 335 & 100 & 800 & 308 & 362 & 130 & $1,065(67)$ & $535(33)$ & $978(61)$ & $622(39)$ \\
\hline 2 & 300 & 152 & 114 & 34 & 600 & 195 & 289 & 116 & $418(70)$ & $182(30)$ & $679(57)$ & $521(43)$ \\
\hline \multicolumn{13}{|l|}{ Yang, 2009 (18) } \\
\hline 1 & 1,013 & 468 & 421 & 124 & 1,131 & 460 & 500 & 171 & $1,357(67)$ & 669 (33) & $1,420(63)$ & $842(37)$ \\
\hline 2 & 827 & 286 & 394 & 147 & 827 & 257 & 383 & 187 & $966(58)$ & $688(42)$ & $897(54)$ & $757(46)$ \\
\hline
\end{tabular}

Table IV. Risk of cancer associated with the genotypes of FEN1 -69G>A (rs174538).

\begin{tabular}{|c|c|c|c|c|c|c|}
\hline \multirow[b]{2}{*}{ Genotype } & \multirow[b]{2}{*}{ OR } & \multirow[b]{2}{*}{$95 \% \mathrm{CI}$} & \multirow[b]{2}{*}{ P-value } & \multicolumn{2}{|c|}{ Heterogeneity } & \multirow{2}{*}{$\begin{array}{c}\text { Effects } \\
\text { model }\end{array}$} \\
\hline & & & & $\mathrm{I}^{2}, \%$ & P-value & \\
\hline AA & 1 (Reference) & & & & & \\
\hline GA & 1.43 & $1.27-1.60$ & $<0.00001$ & 0 & 0.99 & $\mathrm{~F}$ \\
\hline GG & 1.85 & $1.65-2.08$ & $<0.00001$ & 0 & 0.05 & $\mathrm{R}$ \\
\hline $\mathrm{GA}+\mathrm{GG}$ & 1.28 & $1.16-1.42$ & $<0.00001$ & 91 & $<0.00001$ & $\mathrm{R}$ \\
\hline
\end{tabular}

AA genotype was the reference genotype in all analyses. OR, odds ratio; CI, confidence interval; F, fixed effects model; R, random effects model.

Table V. Risk of cancer associated with the genotypes of FEN1 4150G>T (rs4246215).

\begin{tabular}{lcccccr}
\hline Genotype & OR & $95 \% \mathrm{CI}$ & P-value & \multicolumn{2}{c}{ Heterogeneity } & $\begin{array}{r}\text { Effects } \\
\text { model }\end{array}$ \\
\hline TT & 1 (Reference) & & & & P-value & \\
GT & 1.34 & $1.20-1.50$ & $<0.00001$ & 0 & 0.97 & $\mathrm{~F}$ \\
GG & 1.71 & $1.52-1.92$ & $<0.00001$ & 0 & 0.56 & $\mathrm{~F}$ \\
GT+GG & 1.50 & $1.35-1.67$ & $<0.00001$ & 91 & 0.91 & $\mathrm{~F}$ \\
\hline
\end{tabular}

TT genotype was the reference genotype in all analyses. OR, odds ratio; CI, confidence interval; F, fixed effects model; R, random effects model.

models when all the studies were pooled into the meta-analysis. As shown in Table IV, carriers of the FEN1 -69GG genotype showed a significantly elevated risk of cancer compared to
-69AA carriers (OR, 1.85; 95\% CI, 1.65-2.08; P<0.00001). Logistic regression analyses also revealed that individuals with FEN1 -69GA genotypes were significantly associated with 


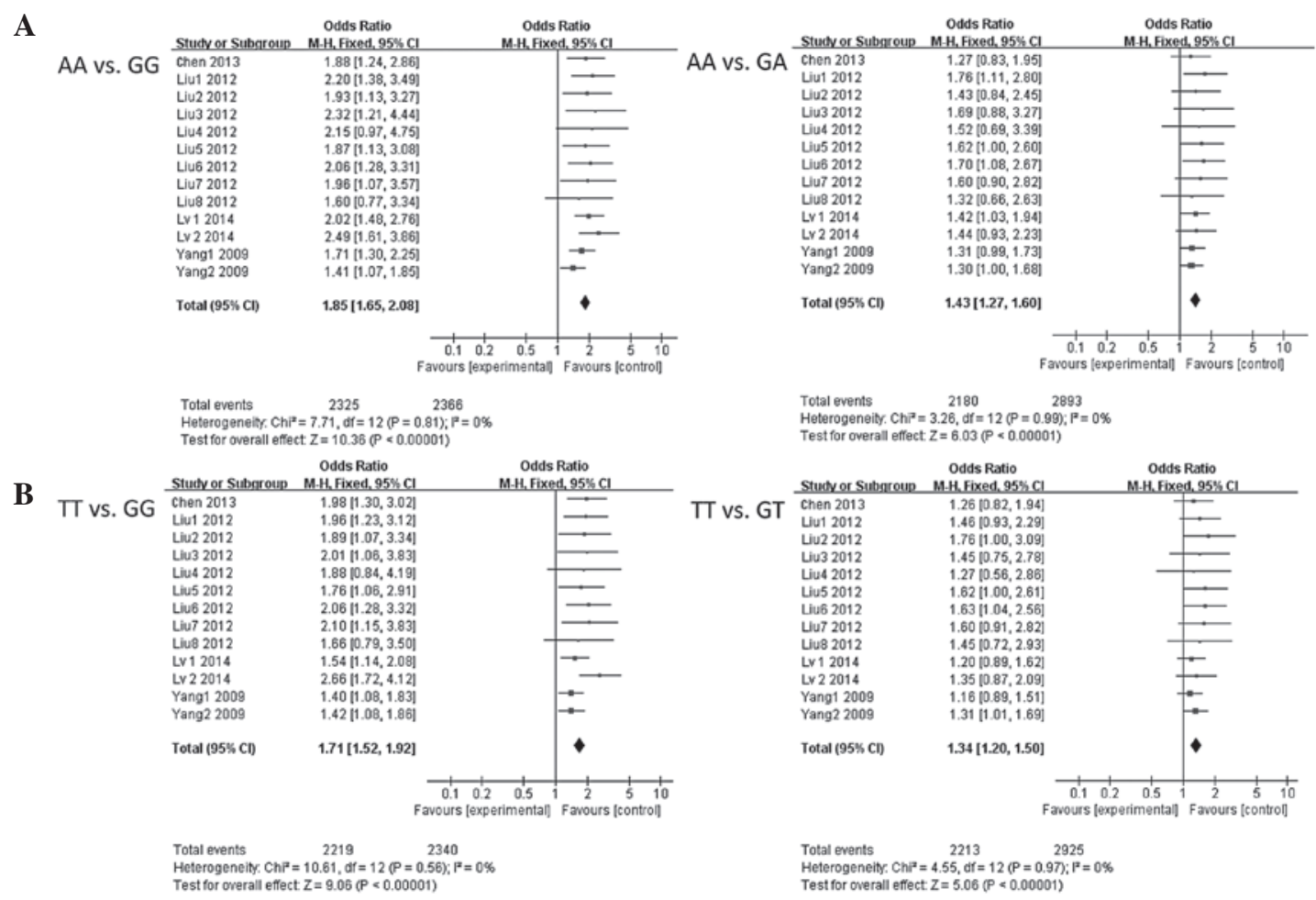

Figure 2. Combined meta-analyses of the associations between the FEN1 polymorphisms and risk of cancer. (A) -69G $>$ A. (B) $4150 \mathrm{G}>\mathrm{T}$. The squares and horizontal lines correspond to the study specific OR and $95 \% \mathrm{CI}$. The area of the squares reflects the weight (inverse of the variance). The diamond represents the summary OR and $95 \%$ CI. OR, odds ratio; CI, confidence interval.

A

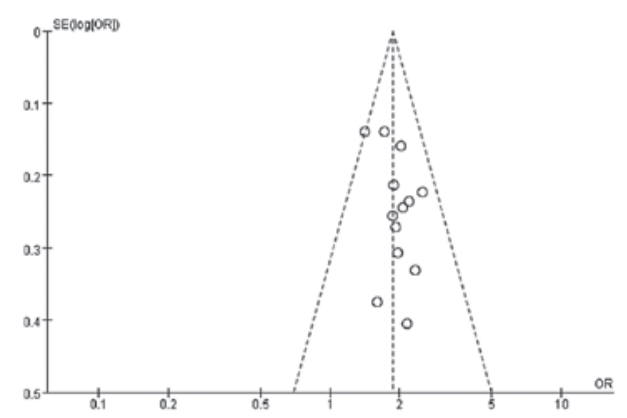

B

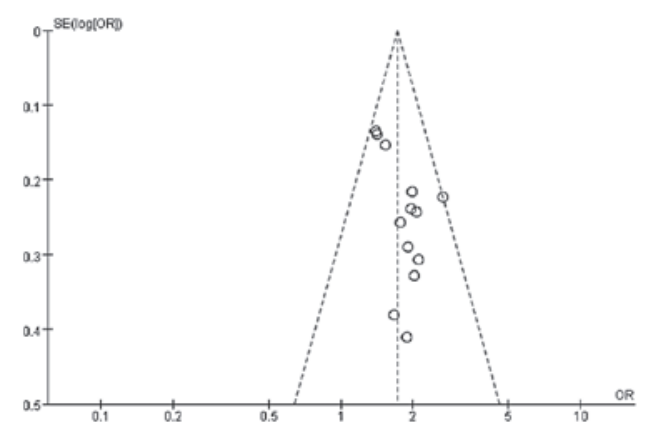

Figure 3. Funnel plot assessing evidence of publication bias from the 13 studies. (A) -69G>A: AA vs. GG. (B) 4150G>T: TT vs. GG. SE, standard error; OR, odds ratio.

increased cancer risk compared to -69AA genotypes (OR, 1.43; 95\% CI, 1.27-1.60; $\mathrm{P}<0.00001)$. In addition, the variant $\mathrm{GG}+\mathrm{GA}$ genotypes were associated with an increased cancer risk when compared to the -69AA genotypes (OR, 1.28; 95\% CI, 1.16-1.42; $\mathrm{P}<0.00001)$. Similar results were observed for the $4150 \mathrm{G}>\mathrm{T}$ polymorphism. As shown in Table V, the FEN1 4150GG genotype showed a significantly elevated risk of cancer compared to 4150TT carriers (OR, 1.71; 95\% CI, 1.52-1.92; $\mathrm{P}<0.00001)$. Logistic regression analyses also revealed that individuals with the FEN1 4150GT genotypes were significantly associated with an increased cancer risk compared to the 4150TT genotypes (OR, 1.34; 95\% CI, 1.20-1.50; P<0.00001). The variant GG+GT genotypes were associated with an increased cancer risk when compared to the 4150TT genotypes (OR, 1.50; 95\% CI, 1.35-1.67; P<0.00001).

Tests of heterogeneity. Statistically significant heterogeneity was observed between trials of the following analyses using the Q statistic. As shown in Fig. 2, for AA vs. GG: $P_{\text {heterogeneity }}=0.05$, $\mathrm{I}^{2}=0$, and therefore, a random effect model was performed; for AA vs. GA: $P_{\text {heterogeneity }}=0.99, \mathrm{I}^{2}=0$, a fixed-effect model was performed; for TT vs. GG: $P_{\text {heterogeneity }}=0.56, \mathrm{I}^{2}=0$, a fixed-effect model was performed; and for TT vs. GT: $P_{\text {heterogeneity }}=0.91$, $\mathrm{I}^{2}=0$, a fixed-effect model was performed.

Publication bias. Begg's funnel plot and Egger's test were performed to assess the publication bias. The funnel plots did not reveal any clear asymmetry in all the genotypes (Fig. 3), and the results of Egger's test revealed no publication bias $(\mathrm{P}>0.05)$.

\section{Discussion}

FEN1 exhibits a prominent role in maintaining genomic stability and protecting against malignant transformation through its involvement in DNA repair and other multiple DNA metabolic pathways (8). Therefore, the structure or 
functional deficiency of FEN1 may destroy the genomic stability to increase the risk of cancer. As previously mentioned, FEN1 mutations reduced nuclease activity to lead to cancer initiation and development (15). FEN1 -69 G and $4150 \mathrm{G}$ alleles, which were correlated to significantly decreased FEN1 mRNA expression in normal gastrointestinal tissues, were associated with increased gastrointestinal cancer risks compared to $-69 \mathrm{~A}$ and $4150 \mathrm{~T}$ alleles in two independent case-control cohorts (21). The FEN1 polymorphisms, rs174538 and rs4246215, may be common cancer risk factors.

In the present meta-analysis, all 13 case-control studies were pooled in a Chinese population to estimate the overall cancer risk of the SNPs. In the present study, the AA and TT genotypes were used as a reference genotype in all the analyses. For rs174538, it was found that individuals exhibiting the GG and GG/GA genotypes were significantly associated with an increased risk of cancer compared to the -69AA genotype. In the combined meta-analyses, the -69GG genotype had a 1.85 -fold increased risk for cancer. Similar results were identified for rs 4246215 , all the genotypes were significantly associated with an increased cancer risk compared to the TT genotype $(\mathrm{P}<0.01)$. The present study indicated that functional rs174538 and rs4246215 were significantly associated with an increased risk of cancer. These results are consistent to the findings in breast cancer, lung cancer, hepatocellular carcinoma, esophageal cancer, gastric cancer, colorectal cancer and glioma from different medical centers of China $(18,20-22)$, which confirmed our speculation that the functional genetic variants in the FENl gene may affect cancer risk as common factors.

When interpreting the results of the present study, certain limitations of the meta-analysis must be considered. Firstly, all the cancer cases and controls were hospital-based, and inherent selection bias may exist. Thus, it is important to validate these findings in a population-based prospective study. Secondly, the meta-analysis was based on pooled data and no individual data was available; thus, the risk of cancer could not be assessed according to stratification of gene-environment and other risk factors of cancer. Thirdly, all the subjects were Chinese, and all the genotyping methods in the included studies were polymerase chain reaction-restriction fragment length polymorphism. Further large-scale multicenter studies with more detailed individual data, with different environmental backgrounds are warranted to further validate the gene-gene and gene-environment interactions on SNPs and cancer risk.

In conclusion, the present meta-analysis provides evidence of the effects of FEN1 SNPs (rs174538 and rs4246215) on the cancer risk. The study indicated that functional rs174538 and rs4246215 were significantly associated with an increased risk of cancer in the Chinese population. Further studies based on different ethnicity are warranted to verify these findings.

\section{Acknowledgements}

The present study was supported by the National Natural Science Foundation of China (grant no. 81471670); the International Cooperative Project of Shaanxi province, China (grant no. 2013KW-32-01); the Fundamental Research Funds for the Central Universities, China; and the Specialized Research Fund of the Second Affiliated Hospital of Xi'an Jiaotong University, China [grant no. RC (GG) 201203].

\section{References}

1. Liu Y, Kao HI and Bambara RA: Flap endonuclease 1: a central component of DNA metabolism. Annu Rev Biochem 73: 589-615, 2004.

2. Zheng L, Jia J, David Finger LD, Guo Z, et al: Functional regulation of FEN1 nuclease and its link to cancer. Nucleic Acid Res 39: 781-794, 2011.

3. Lieber MR: The FEN-1 family of structure-specific nucleases in eukaryotic DNA replication, recombination and repair. Bioessays 19: 233-240, 1997.

4. Tomlinson CG, Atack JM, Chapados B, et al: Substrate recognition and catalysis by flap endonucleases and related enzymes. Biochem Soc Trans 38: 433-437, 2010.

5. Harrington JJ and Lieber MR: The characterization of a mammalian DNA structure-specific endonuclease. EMBO J 13: 1235-1246, 1994.

6. Shen B, Singh P, Liu R, et al: Multiple but dissectible functions of FEN-1 nucleases in nucleic acid processing, genome stability and diseases. Bioessays 27: 717-729, 2005.

7. Reagan MS, Pittenger C, Siede $W$ and Friedberg EC: Characterization of a mutant strain of Saccharomyces cerevisiae with a deletion of the RAD27 gene, a structural homolog of the RAD2 nucleotide excision repair gene. J Bacteriol 177: 364-371, 1995.

8. Zheng L, Zhou M, Chai Q, et al: Novel function of the flap endonuclease 1 complex in processing stalled DNA replication forks. EMBO Rep 6: 83-89, 2005.

9. Zheng L, Dai H, Qiu J, et al: Disruption of the FEN-1/PCNA interaction results in DNA replication defects, pulmonary hypoplasia, pancytopenia and newborn lethality in mice. Mol Cell Biol 27: 3176-3186, 2007.

10. Henneke G, Friedrich-Heineken E and Hubscher U: Flap endonuclease 1: a novel tumour suppresser protein. Trends Biochem Sci 28: 384-390, 2003.

11. Henneke G, Koundrioukoff S and Hubscher U: Phosphorylation of human Fen1 by cyclin-dependent kinase modulates its role in replication fork regulation. Oncogene 22: 4301-4313, 2003.

12. Kucherlapati M, Yang K, Kuraguchi M, et al: Haploinsufficiency of flap endonuclease (Fen1) leads to rapid tumor progression. Proc Natl Acad Sci 99: 9924-9929, 2002.

13. Wu Z, Lin Y, Xu H, et al: High risk of benzo [alpha] pyrene-induced lung cancer in E160D FEN1 mutant mice. Mutat Res 731: 85-91, 2001.

14. Xu H, Zheng L, Dai H, et al: Chemical-induced cancer incidence and underlying mechanisms in Fen1 mutant mice. Oncogene 30: 1072-1081, 2011.

15. Tishkoff DX, Filosi N, Gaida GM and Kolodner RD: A novel mutation avoidance mechanism dependent on S. cerevisiae RAD27 is distinct from DNA mismatch repair. Cell 88: 253-263, 1997.

16. Parrish JZ, Yang CL, Shen BH and Xue D: CRN-1, a Caenorhabditis elegans FEN-1 homologue, cooperates with CPS-6/EndoG to promote apoptotic DNA degradation. EMBO J 22: 3451-3460, 2003.

17. Zheng L, Dai H, Zhou M, et al: Fen1 mutations result in autoimmunity, chronic inflammation and cancers. Nat Med 13: 812-819, 2007.

18. Yang M, Guo H, Wu C, et al: Functional FEN1 polymorphisms are associated with DNA damage levels and lung cancer risk. Hum Mutat 30: 1320-1328, 2009.

19. Dai ZJ, Wang XJ, Kang AJ, et al: Association between APE1 single nucleotide polymorphism (rs1760944) and cancer risk: a meta-analysis based on 6,419 cancer cases and 6,781 case-free controls. J Cancer 5: 253-259, 2014.

20. Lv Z, Liu W, Li D, et al: Association of functional FEN1 genetic variants and haplotypes and breast cancer risk. Gene 538: 42-45, 2014.

21. Chen YD, Zhang X, Qiu XG, et al: Functional FEN1 genetic variants and haplotypes are associated with glioma risk. J Neurooncol 111: 145-151, 2013.

22. Liu L, Zhou C, Zhou L, et al: Functional FEN1 genetic variants contribute to risk of hepatocellular carcinoma, esophageal cancer, gastric cancer and colorectal cancer. Carcinogenesis 33: 119-123, 2012. 